Olivera Iskrenovic-Momcilovic

https://doi.org/10.21278/TOF.42402

ISSN 1333-1124

eISSN 1849-1391

\title{
DISCRETE-TIME SLIDING MODE CONTROL WITH INTEGRAL COMPENSATION OF OUTPUT ERROR
}

\begin{abstract}
Summary
In this paper, a simple new design method of the sliding mode control based on the integral compensation of an output error is described. The key to this method is to obtain a control with a switching function. The proposed linear control input is robust against plant parameter deviations and external disturbances. We confirmed the effectiveness of the proposed method through simulation of a second and a third order plant.
\end{abstract}

Key words: $\quad$ control, discrete-time, integral, sliding mode, variable structure system.

\section{Introduction}

Sliding mode control (SMC) is a term used in the context of variable structure systems [1]. Variable structure system (VSS) is basically a set of arbitrary numbers of continuous subsystems, which are alternately included in a certain logic, resulting in control, which is basically a discontinuous function of a variable state and an external disturbance. Which of the structures will be activated at some point is determined by the fault of the switch, whose definition is the main task of the sliding mode control system. If the switching curve is chosen for the deviation from the actual to the desired system motion trajectory, then the basic idea of the sliding mode is to bring the actual trajectory of the system to the desired trajectory in the state space and to retain it, regardless of the influence of the external disturbance and the change in the system parameters.

The basic characteristic of a system in sliding mode is its robustness against changes in parameters and external disturbance $[2,3]$, as well as the possibility of applying the sliding mode to a large number of non-linear systems. Although the sliding mode algorithm is basically simple, the inability of real physical switches to overlap at a certain moment leads to a phenomenon known as "chattering". Chattering refers to a high frequency signal and a final amplitude that oscillates around the equilibrium point (state of the system) or around the switching curve. The phenomenon of chattering is not only a serious problem for the control signal as the desired dynamic characteristics of the system cannot be achieved, but it also a destructive effect on the executive body, as well as on other physical parts of the system [4].

SMC was first proposed in the early 1960s by Emelyanov [5] and was later popularized by Itkis and Utkin [6-8]. Bartolini and other researchers [10-12] later applied SMC to multiinput and multi-input multi-output systems. Utkin and Lee [13] were dealing with the problem of chattering caused by control input switching. Choi et al. $[14,15]$ proposed SMC with a 
moving sliding surface, which was designed to pass arbitrary initial conditions, and subsequently move towards a predetermined sliding surface by rotating and/or shifting. Chern et al. [16-18] proposed a variable structure controller design with integral compensation for different systems: induction motor, synchronous generator and electrohydraulic velocity servosystems. Integral-type SMC that can maintain the system state at all times has been proposed by several researchers [19-25]. In addition, some studies presented techniques to remove the chattering phenomenon [26-28].

All previous papers consider integral-type SMC in the continual domain. This paper proposes SMC based on the integral compensation of an output error in the discrete-time domain. The basic idea is to design a proper switching hyperplane so that the sliding mode starts from the initial time instant. As a result, the robustness of the system can be guaranteed from the beginning of the process and the reaching phase is eliminated. The obtained SMC has many advantages such as fast response, invariance to plant parameter deviations and ability to compensate for external disturbances.

\section{Representation of a plant in discrete-time state space}

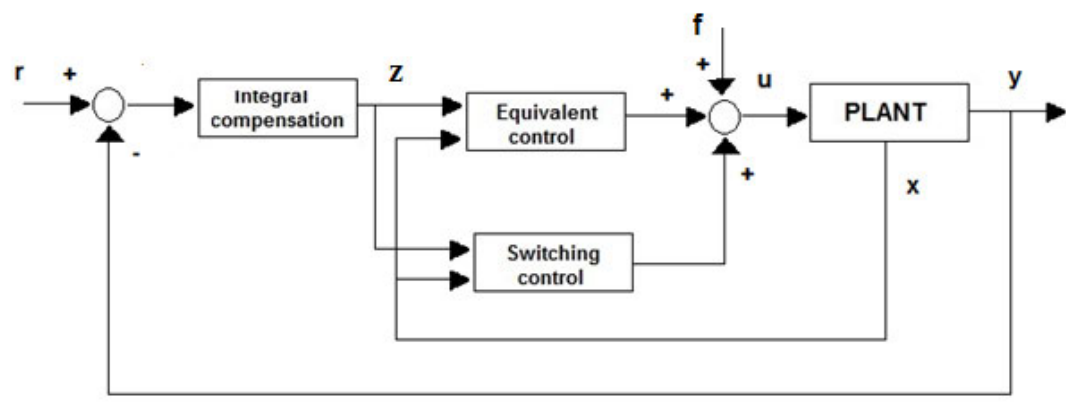

Fig. 1 Sliding mode control with output error integration

Let the plant (Fig. 1) be defined as follows:

$$
W_{p}(s)=\frac{b}{s^{n}+a_{n-1} s^{n-1}+\ldots+a_{1} s+a_{0}}
$$

where:

$$
b, a_{i}(i=1,2, \ldots n-1) \text { - plant parameters. }
$$

The system using SMC with the integral compensation of an output error is described as follows:

$$
\begin{aligned}
& \dot{\mathbf{x}}(t)=\mathbf{A} \mathbf{x}(t)+\mathbf{b} u(t) \\
& y(t)=x_{1}(t) \\
& z(t)=\int(r(t)-y(t)) d t
\end{aligned}
$$

where:

$$
\begin{aligned}
& \mathbf{x}(t) \in \mathfrak{R}^{n} \text { - state vector, } u(t) \in \mathfrak{R} \text { - control signal, } \\
& r(t) \in \mathfrak{R} \text { - reference signal, } y(t) \in \mathfrak{R} \text { - controlled output, } \\
& z(t) \in \mathfrak{R} \text { - integral of output error, }
\end{aligned}
$$




$$
\mathbf{A}=\left[\begin{array}{ccccc}
0 & 1 & 0 & \ldots & 0 \\
0 & 0 & 1 & \ldots & 0 \\
\ldots & \ldots & \ldots & \ldots & \ldots \\
0 & 0 & 0 & \ldots & 1 \\
-a_{0} & -a_{1} & -a_{2} & \ldots & -a_{n-1}
\end{array}\right] \quad \mathbf{b}=\left[\begin{array}{c}
0 \\
0 \\
\ldots \\
0 \\
b
\end{array}\right] \text { - plant matrices }
$$

Eq. (2) can be rewritten in a matrix form as follows:

$$
\dot{\mathbf{x}_{m}}(t)=\mathbf{A}_{m} \mathbf{x}_{m}(t)+\mathbf{b}_{m} u(t)+\mathbf{d} r(t)
$$

where:

$$
\mathbf{x}_{m}(t)=\left[\begin{array}{c}
z(t) \\
x_{1}(t) \\
x_{2}(t) \\
\ldots \\
x_{n-1}(t) \\
x_{n}(t)
\end{array}\right] \quad \mathbf{A}_{m}=\left[\begin{array}{cccccc}
0 & -1 & 0 & 0 & \ldots & 0 \\
0 & 0 & 1 & 0 & \ldots & 0 \\
0 & 0 & 0 & 1 & \ldots & 0 \\
\ldots & \ldots & \ldots & \ldots & \ldots & \ldots \\
0 & 0 & 0 & 0 & \ldots & 1 \\
0 & -a_{0} & -a_{1} & -a_{2} & \ldots & -a_{n-1}
\end{array}\right] \quad \mathbf{b}_{m}=\left[\begin{array}{c}
0 \\
0 \\
0 \\
\ldots \\
0 \\
b
\end{array}\right] \quad \mathbf{d}=\left[\begin{array}{c}
1 \\
0 \\
0 \\
\ldots \\
0 \\
0
\end{array}\right]
$$

This modified model of the plant can be realized by a computer (discretely). An equivalent discrete-time model of the system (3) is [19]:

$$
\delta \mathbf{x}_{\mathbf{m}}(k)=\mathbf{A}_{\boldsymbol{\delta}} \mathbf{x}_{\mathbf{m}}(k)+\mathbf{b}_{\boldsymbol{\delta}} u(k)+\mathbf{d}_{\boldsymbol{\delta}} r(k)
$$

where:

$$
\mathbf{A}_{\boldsymbol{\delta}}=\frac{e^{\mathbf{A} T}-\mathbf{I}}{T} \quad \mathbf{b}_{\boldsymbol{\delta}}=\frac{1}{T} \int_{0}^{T} e^{\mathbf{A} \tau} \mathbf{b} d \tau \quad \mathbf{d}_{\boldsymbol{\delta}}=\frac{1}{T} \int_{0}^{T} e^{\mathbf{A} \tau} \mathbf{d} d \tau
$$

The expression for $\delta \mathbf{x}_{\mathbf{m}}(k)$ represents the first differential:

$$
\delta \mathbf{x}_{\mathbf{m}}(k)=\frac{\mathbf{x}_{\mathbf{m}}(k+1)-\mathbf{x}_{\mathbf{m}}(k)}{T}
$$

\section{Design of discrete-time sliding mode control}

The discrete-time SMC is expressed as

$$
\delta \mathbf{x}_{\mathbf{m}}(k)=\mathbf{A}_{\boldsymbol{\delta}} \mathbf{x}_{\mathbf{m}}(k)+\mathbf{b}_{\boldsymbol{\delta}} u(k)+\mathbf{d}_{\boldsymbol{\delta}} r(k) \quad g(k)=\mathbf{c}_{\boldsymbol{\delta}} \mathbf{x}_{\mathbf{m}}(k)
$$

where:

$$
\begin{aligned}
& g(k) \in \mathfrak{R} \text { - switching hyperplane } \\
& \mathbf{c}_{\boldsymbol{\delta}} \in \mathfrak{R} \text { - switching matrix }
\end{aligned}
$$

First, to realize the control using a sliding mode controller, we are required to design a switching matrix $\mathbf{c}_{\boldsymbol{\delta}}$. The switching matrix $\mathbf{c}_{\boldsymbol{\delta}}$ is determined by the solution $\mathbf{P}$ of the discretetime Riccati equation as

$$
\mathbf{c}_{\delta}=\mathbf{b}_{\delta} \mathbf{P}
$$

The discrete-time Ricatti equation is given as

$$
\mathbf{A}_{\delta}^{\mathbf{T}} \mathbf{P} \mathbf{A}_{\delta}-\mathbf{P}-\mathbf{A}_{\delta}^{\mathbf{T}} \mathbf{P} \mathbf{b}_{\delta}\left(\mathbf{b}_{\delta}^{\mathbf{T}} \mathbf{P} \mathbf{b}_{\delta}+\mathbf{P}\right)^{-1} \mathbf{b}_{\delta}^{\mathbf{T}} \mathbf{P} \mathbf{A}_{\delta}+\mathbf{Q}=0
$$


where:

Q - diagonal matrix with positive elements.

We wish to design a DTVS controller for the system (4), so that the control is

$$
u(k)=u_{e q}(t)+u_{s w}(t)
$$

where:

$$
\begin{aligned}
& u_{e q}(k) \text { - equivalent control } \\
& u_{s w}(k) \text { - switching control }
\end{aligned}
$$

The equivalent control $u_{e q}(k)$ is determined from the conditions [8] that the system remains on the switching hyper plane

$$
g(k+1)=g(k) \text { for e ach } k \text { or } g(k)=0, \quad \delta g(k)=0
$$

The first difference $\delta g(k)$ of the expression (6) is

$$
\delta g(k)=\mathbf{c}_{\boldsymbol{\delta}} \mathbf{A}_{\boldsymbol{\delta}} \mathbf{x}_{\mathbf{m}}(k)+\mathbf{c}_{\boldsymbol{\delta}} \mathbf{b}_{\boldsymbol{\delta}} u(k)+\mathbf{c}_{\boldsymbol{\delta}} \mathbf{d}_{\boldsymbol{\delta}} r(k)
$$

Based on the relations (8) and (9), the equivalent control $u_{e q}(k)$ is

$$
u_{e q}(k)=-\left(\mathbf{c}_{\boldsymbol{\delta}} \mathbf{b}_{\boldsymbol{\delta}}\right)^{-1}\left(\mathbf{c}_{\boldsymbol{\delta}} \mathbf{A}_{\boldsymbol{\delta}} \mathbf{x}_{\mathbf{m}}(k)+\mathbf{c}_{\boldsymbol{\delta}} \mathbf{d}_{\boldsymbol{\delta}} r(k)\right)
$$

The switching control $u_{s w}(k)$ is used for reaching the state variable to the switching hyperplane and remain on it. It is designed as nonlinear control as

$$
u_{s w}(k)=-k\left(\mathbf{c}_{\boldsymbol{\delta}} \mathbf{b}_{\boldsymbol{\delta}}\right)^{-1} \frac{g(k)}{|g(k)|}
$$

Based on the relations (10) and (11), the control is presented as follows

$$
u(k)=-\left(\mathbf{c}_{\boldsymbol{\delta}} \mathbf{b}_{\boldsymbol{\delta}}\right)^{-1}\left(\mathbf{c}_{\boldsymbol{\delta}} \mathbf{A}_{\boldsymbol{\delta}} \mathbf{x}_{\mathbf{m}}(k)+\mathbf{c}_{\boldsymbol{\delta}} \mathbf{d}_{\boldsymbol{\delta}} r(k)\right)-k\left(\mathbf{c}_{\boldsymbol{\delta}} \mathbf{b}_{\boldsymbol{\delta}}\right)^{-1} \frac{g(k)}{|g(k)|}
$$

Futura [29] defined the existence and reachability conditions of a sliding mode on the switching hyperplane $g(k)=0$ in the following form:

$$
\delta V(k)<0 \Leftrightarrow V(k+1)-V(k)<0
$$

where:

$$
V(k)=\frac{1}{2}[g(k)]^{2}-\text { Lyapunov function }
$$

The first difference $\delta V(k)$ of the expression (14) is

$$
\begin{aligned}
\delta V(k)= & g(k) \delta g(k)=g(k) \mathbf{c}_{\boldsymbol{\delta}}\left(\mathbf{A}_{\boldsymbol{\delta}} \mathbf{x}_{\mathbf{m}}(k)+\mathbf{b}_{\boldsymbol{\delta}} u(k)+\mathbf{d}_{\boldsymbol{\delta}} r(k)\right) \\
= & g(k)\left[\mathbf{c}_{\boldsymbol{\delta}} \mathbf{A}_{\boldsymbol{\delta}} \mathbf{x}_{\mathbf{m}}(k)-\mathbf{c}_{\boldsymbol{\delta}} \mathbf{b}_{\boldsymbol{\delta}}\left\{( \mathbf { c } _ { \boldsymbol { \delta } } \mathbf { b } _ { \boldsymbol { \delta } } ) ^ { - 1 } \left(\mathbf{c}_{\boldsymbol{\delta}} \mathbf{A}_{\boldsymbol{\delta}} \mathbf{x}_{\mathbf{m}}(k)+\mathbf{c}_{\boldsymbol{\delta}} \mathbf{d}_{\boldsymbol{\delta}} r(k)\right.\right.\right. \\
& \left.\left.-K\left(\mathbf{c}_{\boldsymbol{\delta}} \mathbf{b}_{\boldsymbol{\delta}}\right)^{-1} \frac{g(k)}{|g(k)|}\right\}+\mathbf{c}_{\boldsymbol{\delta}} \mathbf{d}_{\boldsymbol{\delta}} r(k)\right]
\end{aligned}
$$


Based on the relations (13) and (15), the existence and reachability conditions of a sliding mode are $K>0$. If the control is designed by using Eq.12, a chattering phenomenon occurs. When $\delta g(k)=0$ for $u_{s w}(k),|g(k)|$ in the denominator becomes 0 , quick switching of the input occurs. This phenomenon is called chattering, which refers to a high-frequency vibrations. To avoid this problem, the switching control $u_{s w}(k)(11)$ is modified as follows:

$$
u_{s w}(k)=-k\left(\mathbf{c}_{\boldsymbol{\delta}} \mathbf{b}_{\boldsymbol{\delta}}\right)^{-1} \frac{g(k)}{|g(k)|+\alpha}
$$

where:

$$
\alpha \in \mathfrak{R}, \quad \alpha>0 \text { - arbitrary positive constant }
$$

Eq. 16 becomes a smooth function and the chattering phenomenon can be relieved. In total, the control is presented by the sum of the equivalent (10) and switching (16) control as

$$
u(k)=-\left(\mathbf{c}_{\boldsymbol{\delta}} \mathbf{b}_{\boldsymbol{\delta}}\right)^{-1}\left(\mathbf{c}_{\boldsymbol{\delta}} \mathbf{A}_{\boldsymbol{\delta}} \mathbf{x}_{\mathbf{m}}(k)+\mathbf{c}_{\boldsymbol{\delta}} \mathbf{d}_{\boldsymbol{\delta}} r(k)\right)-k\left(\mathbf{c}_{\boldsymbol{\delta}} \mathbf{b}_{\boldsymbol{\delta}}\right)^{-1} \frac{g(k)}{|g(k)|+\alpha}
$$

\section{Illustrative example}

a) Example of the control of a second order plant

In order to verify the obtained relations for the synthesis of SMC, the building control of a second order plant, the discrete-time SMC was designed and simulated on a PC control facility, whose transfer function is as follows:

$$
W_{P}(s)=\frac{b}{s^{2}+a_{1} s+a_{0}}, \quad 15 \leq a_{0} \leq 25, \quad 10 \leq a_{1} \leq 20, \quad 60 \leq b \leq 80 . \quad f(t)=0.1
$$

The system using SMC with the integral compensation of an output error (3) is described as follows:

$$
\dot{\mathbf{x}}_{m}(t)=\left[\begin{array}{ccc}
0 & -1 & 0 \\
0 & 0 & 1 \\
0 & -20 & -15
\end{array}\right] \mathbf{x}_{m}(t)+\left[\begin{array}{c}
0 \\
0 \\
70
\end{array}\right] u(t)+\left[\begin{array}{l}
1 \\
0 \\
0
\end{array}\right] r(t)
$$

The discrete model of the system (17) for the sampling period $T=1 m s$, according to (5) has the following form:

$$
\delta \mathbf{x}_{\mathbf{m}}(k)=\left[\begin{array}{ccc}
0 & -0.9996 & 0.002 \\
0 & 0.002 & 0.9998 \\
0 & -19.992 & -14.996
\end{array}\right] \mathbf{x}_{\mathbf{m}}(k)+\left[\begin{array}{c}
0 \\
0.002 \\
69.994
\end{array}\right] u(k)+\left[\begin{array}{c}
0.9998 \\
0.001 \\
0
\end{array}\right] r(k)
$$

Let us give the diagonal matrix

$$
\mathbf{Q}=\left[\begin{array}{lll}
1 & 0 & 0 \\
0 & 1 & 0 \\
0 & 0 & 1
\end{array}\right]
$$

then the switching matrix $\mathbf{c}_{\boldsymbol{\delta}}$ is

$$
\mathbf{c}_{\boldsymbol{\delta}}=\left[\begin{array}{lll}
-456.47 & 51.92 & 1.567
\end{array}\right]
$$

For the realization of the discrete-time SMC the following values of parameters can be selected:

$$
k=50, \quad \alpha=0.1
$$


Based on the selected parameters of the discrete-time SM, the simulation results are presented in the form of a diagram of the nominal plant response (Fig. 2, Fig. 3) for $r=0$ control (Fig. 4) and switching hyperplane (Fig. 5). The system has good properties of eliminating external disturbances (Fig. 2, Fig. 3) and compensation for changes of plant parameters (Fig. 3).

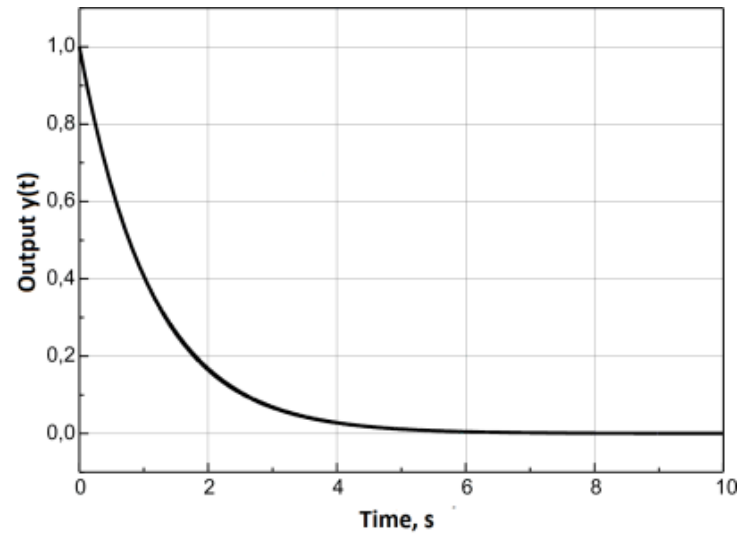

Fig. 2 Response of nominal plant

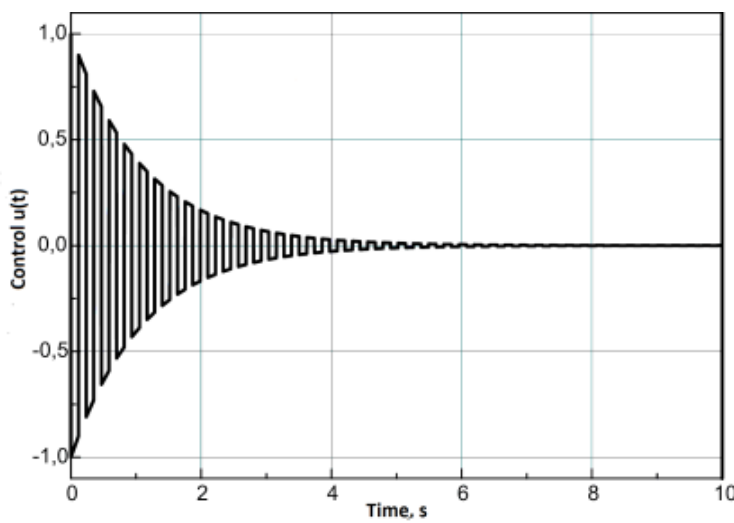

Fig. 4 Control of nominal plant

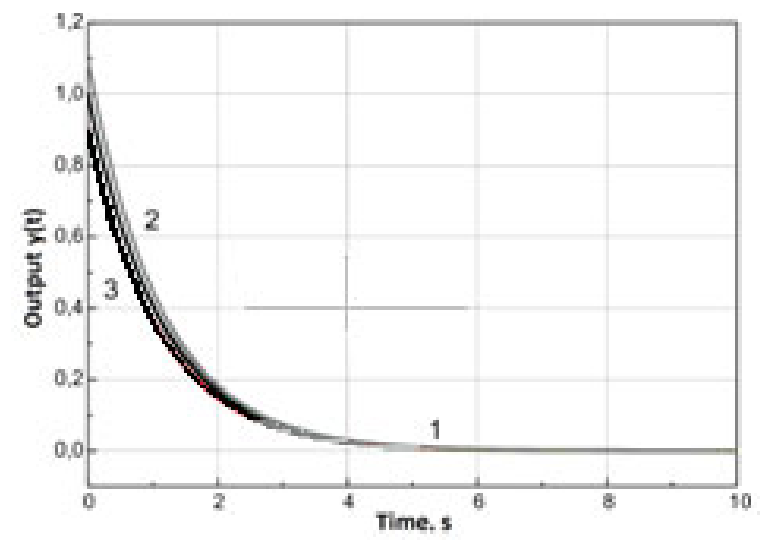

Fig. 3 Plant response to different parameters values

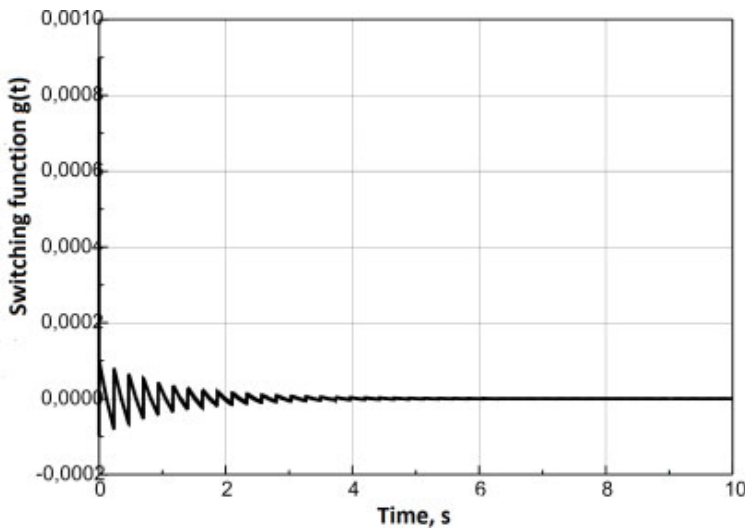

Fig. 5 Switching hyperplane of nominal plant

\section{b) Example of the control of a higher order plant}

In order to validate the proposed method, discrete-time SMC is designed and simulated on a PC to control a third order plant:

$$
W_{P}(s)=\frac{20}{s^{3}+a_{2} s^{2}+a_{1} s+a_{0}}, 15 \leq a_{0}=a_{2} \leq 25,110 \leq a_{1} \leq 130,10 \leq b \leq 30, f(t)=0.1
$$

The system using SMC with the integral compensation of an output error (3) is described as follows:

$$
\dot{\mathbf{x}}_{m}(t)=\left[\begin{array}{cccc}
0 & -1 & 0 & 0 \\
0 & 0 & 1 & 0 \\
0 & 0 & 0 & 1 \\
0 & -20 & -120 & -20
\end{array}\right] \mathbf{x}_{m}(t)+\left[\begin{array}{c}
0 \\
0 \\
0 \\
20
\end{array}\right] u(t)+\left[\begin{array}{l}
1 \\
0 \\
0 \\
0
\end{array}\right] r(t)
$$

The discrete model of the system (18) for the sampling period $T=1 \mathrm{~ms}$, according to (5) has the following form: 


$$
\delta \mathbf{x}_{\mathbf{m}}(k)=\left[\begin{array}{cccc}
0 & -0.9996 & 0.002 & 0.002 \\
0 & 0.002 & 0.9998 & 0.001 \\
0 & 0.001 & 0.015 & 0.9999 \\
0 & -19.992 & -119.996 & -19.992
\end{array}\right] \mathbf{x}_{\mathbf{m}}(k)+\left[\begin{array}{c}
0 \\
0.002 \\
0.001 \\
19.994
\end{array}\right] u(k)+\left[\begin{array}{c}
0.9998 \\
0.001 \\
0.001 \\
0
\end{array}\right] r(k)
$$

Let us give the diagonal matrix

$$
\mathbf{Q}=\left[\begin{array}{llll}
1 & 0 & 0 & 0 \\
0 & 1 & 0 & 0 \\
0 & 0 & 1 & 0 \\
0 & 0 & 0 & 1
\end{array}\right]
$$

then the switching matrix $\mathbf{c}_{\tilde{\delta}}$ is

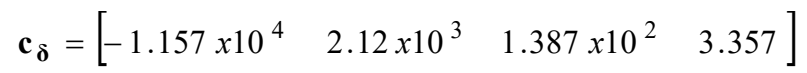

For the realization of the discrete time SMC the following values of parameters can be selected:

$$
k=50, \quad \alpha=0.1
$$

The results of the computer simulation are shown in the form of a diagram of the step response of the plant (Fig. 6 and Fig. 7), control (Fig. 8) and switching hyperplane (Fig. 9). From the obtained diagrams, it is obvious that the system meets all the requirements. It is robust when values of the parameters of the plant are changed within the given boundaries (Fig. 7). It also has good properties of eliminating external disturbances (Fig. 6, Fig. 7).

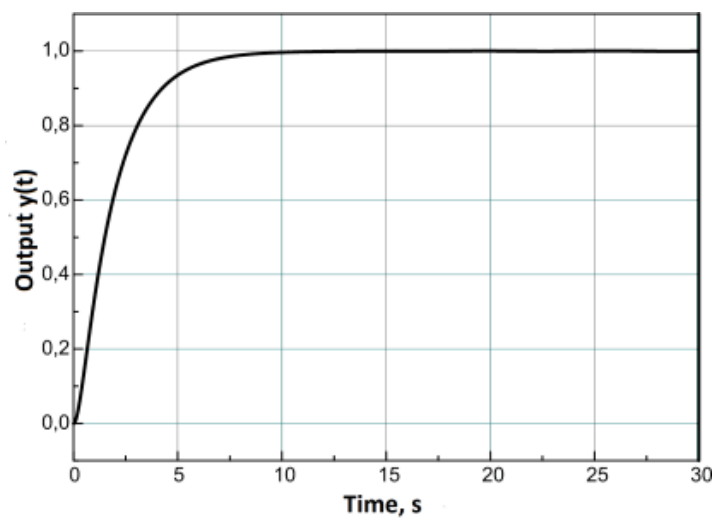

Fig. 6 Step response of nominal plant

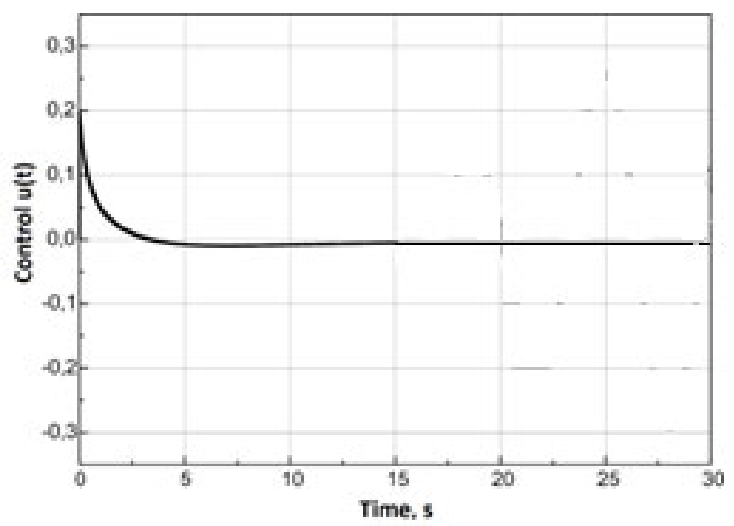

Fig. 8 Control of nominal plant

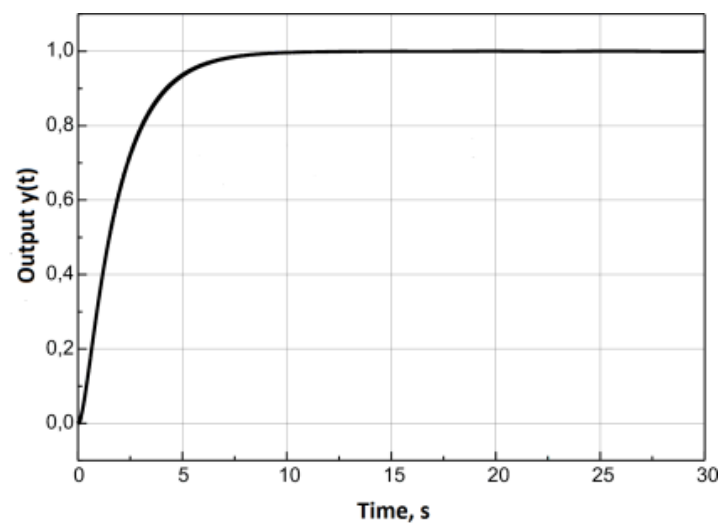

Fig. 7 Plant response to different parameters values

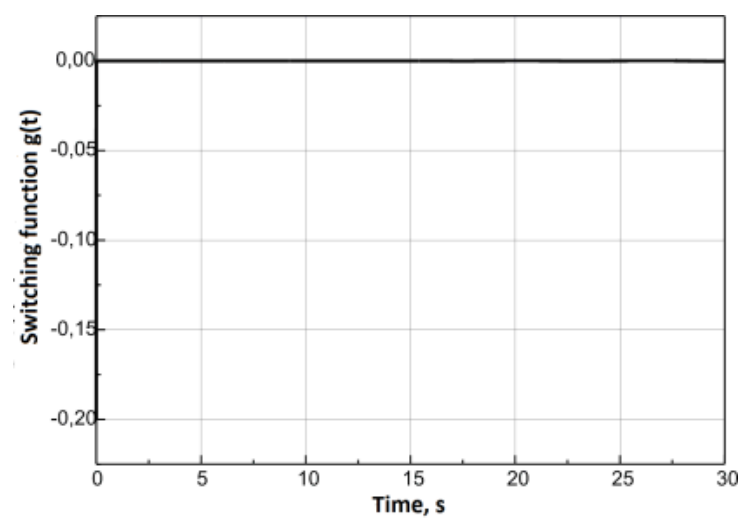

Fig. 9 Switching hyperplane of nominal plant 


\section{Conclusion}

In this paper, a new discrete-time SMC with the integral compensation of an output error is proposed. The design of an optimal switching matrix is described that does not depend on plant parameters. The simulation results show that the proposed method is more effective in the nominal plant, the plant with disturbances, and the plant with parameter deviations. The proposed method is robust against plant parameter deviations and external disturbances.

\section{REFERENCES}

[1] X. Yu, "Sliding-mode control with soft computing: A survey", IEEE Transactions on Industrial Electronics., vol. 56, no. 9, pp. 3275-3285, 2009. https://doi.org/10.1109/TIE.2009.2027531

[2] W. Perroquetti, and J.P. Barbot, Sliding Mode Control In Engineering. Marcel Dekker, Basel, 2002.

[3] K.D. Young, V.I. Utkin, and U. Ozguner, "A control engineer's guide to sliding mode control", In Proceedings of International Workshop of Variable Structure Systems VSS '96, Tokyo, Japan, 1996. https://doi.org/10.1109/VSS.1996.578521

[4] H. Lee, and V.I. Utkin, "Chattering suppression methods in sliding mode control systems", Annual Reviews in Control, vol. 31, no. 2, pp. 179-188, 2007. https://doi.org/10.1016/j.arcontrol.2007.08.001

[5] S.V. Emelyanov, Variable Structure Control Systems, (in Russian) Moscow, Nauka, also Oldburg Verlag Munchen, Wien (in German), 1967.

[6] U. Itkis, Control Systems of Variable Structure, Wiley, New York, 1976.

[7] V.I. Utkin, Sliding Mode and Their Applications, Mir, Moscow, 1978.

[8] V. I. Utkin, "Variable Structure Systems with the Sliding Mode; A survey", IEEE Transaction on Automatic Control, vol. AC-22, no. 2, pp. 212-222, 1977. https://doi.org/10.1109/TAC.1977.1101446

[9] G. Bartolini, E. Punta, and T. Zolezzi, "Simplex sliding mode methods for chattering reduction control of multiinput nonlinear uncertain systems", Automatica, vol. 45, no 8, pp. 1923-1928, 2009. https://doi.org/10.1016/j.automatica.2009.04.014

[10] R.M. Hirschorn, "Generalized sliding-mode control for multi-input nonlinear systems", IEEE Transactions on automatic control, vol. 51, no. 9, pp. 1410-1422, 2006. https://doi.org/10.1109/TAC.2006.880959

[11] Y. Chen, and Y. Chang, "Design of sliding mode controller for MIMO system", In Proceedings.of 8th Asian Control Conference, Kaohsiung, Taiwan. pp. 613-616, 2011.

[12] Jeang-Lin Chang, "Discrete sliding mode control of MIMO linear systems", Asian Journal of Control, vol. 4, no. 2, pp. 217-222, 002.

[13] V. I. Utki, and H. Lee, "Chattering problem in sliding mode control systems", In Proceedings of International Workshop on Variable Structure Systems VSS'06, Alghero, Italy, pp. 346-350, 2006. https://doi.org/10.1109/VSS.2006.1644542

[14] S.B. Choi, and D.W. Park, "Moving sliding surfaces for fast tracking control of second-order dynamical systems", ASME Journal of Dynamic systems, Measurement, and Control, vol. 116, no.1, pp. 154-158, 1993. https://doi.org/10.1115/1.2900671

[15] S.B. Choi, and D.W. Park, "Moving sliding surfaces for high-order variable structure systems", International Journal of Control, vol. 72, no. 11, pp. 960-970, 1999. https://doi.org/10.1080/002071799220506

[16] T.L. Chern, J. Chang, and K.L. Tsai, "Integral-variable-structure-control-based adaptive speed estimator and resistance identifier for an induction motor", International Journal of Control, vol. 69. No. 1, pp. 3147, 1998. https://doi.org/10.1080/002071798222910

[17] G.K. Chang, and T.L. Chern, "Modified integral variable structure model following control of synchronous generator", In Proceedings of American Control Conference, Arlington, USA, pp. 823-828, 2001.

[18] T.L. Chern, and Y.C. Wu, "An optimal variable structure control with integral compensation for electrohydraulic position servo control systems", IEEE Trans. on Industrial Electronics, vol. 39, no.5, pp. 460-463, October 1992. https://doi.org/10.1109/41.161478

[19] Q. Shen, D. Wang, and S. Zhu, "Integral-type sliding mode fault-tolerant control for attitude stabilization of spacecraft", IEEE Transactions on Control Systems Technology, vol. 23, no. 3, pp. 1131-1138, 2015. https://doi.org/10.1109/TCST.2014.2354260 
[20] M. Furat, and I. Eker, "Second-order integral sliding-mode control with experimental application", ISA Transaction, vol. 53, no. 5, pp. 1661-1669, 2014. https://doi.org/10.1016/j.isatra.2014.05.030

[21] Q. Hu, and C.B. Xiao, "Adaptive fault tolerant control using integral sliding mode strategy with application to flexible spacecraft", International Journal of Systems Science, vol. 44, no. 22, pp. 2273 2286, 2013. https://doi.org/10.1080/00207721.2012.702236

[22] A. Benamor, L. Chrifi-alaui, H. Messaoud, and M. Chaabane, "Sliding mode control with integrator for a class of MIMO nonlinear systems", Engineering, vol. 3, pp. 435-444, 2011. https://doi.org/10.4236/eng.2011.35050

[23] Q. Hu, Y. Zhang, Z. Huo, and B. Xia, "Adaptive integral-type sliding mode control for spacecraft attitude maneuvering under actuator stuck failures", Chinese Journal of Aeronautics, vol. 24, no. 1, pp. 32-45, 2011. https://doi.org/10.1016/S1000-9361(11)60005-8

[24] S.C. Tan, Y.M. Lai, C.K. Tse, and C.K. Wu, "A double-integral type of indirect sliding mode controllers for power converters", In Proceedings of IEEE Annual Power Electronics Specialists Conference, Orlando, USA, pp. 177-183, 2007. https://doi.org/10.1109/PESC.2007.4341985

[25] W. Song, Y. Liu, and L. Sun, "Model reference adaptive integral-type sliding mode control design for a class of uncertain systems", In Proceedings of the 6th World Congress of Intelligent Control and Automation, Dalian, China, 2006.

[26] A. Chalanga, S. Kamal, and B. Bandyopadhyay, "Continuous integral sliding mode control: A chattering free approach", In Procedings of IEEE International Symposium on Industrial Electronics (ISIE), Taipei, Taiwan, 2013. https://doi.org/10.1109/ISIE.2013.6563756

[27] Q. Khan, A.I. Bhatti, and Q. Ahmed, "Dynamic integral sliding mode control of nonlinear SISO systems with states dependent matched and mismatched uncertainties", In Procedings of 18th IFAC World Congress, Milano, Italy, pp. 3832-3837, 2011. https://doi.org/10.3182/20110828-6-IT-1002.00068

[28] A. Kuzu, S. Bogosyan, and M. Gokasan, "Comparative evaluation of two chattering-free sliding mode controllers for the control of MEM optical switches", In Procedings of IEEE International Symposium on Industrial Electronics (ISIE), Bari, Italy, pp. 415-421, 2010. https://doi.org/10.1109/ISIE.2010.5637863

[29] K. Furuta. "Sliding mode control of a discrete system", Systems \& Control Letters, vol.14, pp. 145-152. 1990. https://doi.org/10.1016/0167-6911(90)90030-X

Submitted: $\quad 23.6 .2018$

Accepted: $\quad 11.10 .2018$
Olivera Iskrenovic-Momcilovic

University of Novi Sad

Faculty of Education

Novi Sad, Serbia 